\title{
Pathogen Disgust Predicts Stigmatization of Individuals with Mental Health Conditions
}

\author{
Eugene J. Dawydiak ${ }^{1}$ (I) $\cdot$ Holly E. Stafford ${ }^{1} \cdot$ Judith L. Stevenson ${ }^{1} \cdot$ Benedict C. Jones ${ }^{2}$
}

Published online: 17 August 2019

(C) The Author(s) 2019

\begin{abstract}
Both avoidance of individuals with cues of infectious illnesses and stigmatization of other types of individual (e.g., obese individuals) are hypothesized to reflect infectious disease avoidance. However, direct empirical tests of this hypothesis have been somewhat rare. Consequently, we tested for possible relationships between subscales of the Three Domain Disgust Scale and stigmatization of individuals with one of three mental health conditions (schizophrenia, skin-picking disorder, or sexual sadism disorder) in a sample of 117 participants. Scores on the pathogen disgust subscale of the Three Domain Disgust Scale were positively correlated with stigmatization of these mental health conditions. By contrast, scores on the sexual and moral disgust subscales of the Three Domain Disgust Scale were not significantly related to the stigmatization of mental health conditions. When analyzed separately, there were significant positive effects of pathogen disgust for skin picking and sexual sadism, but not schizophrenia. These results potentially implicate overgeneralization of infectious disease avoidance in the stigmatization of individuals with mental health conditions.
\end{abstract}

Keywords Disgust $\cdot$ Pathogens $\cdot$ Stereotypes $\cdot$ Stigma

\section{Introduction}

People tend to avoid stigmatized individuals, such as those with prominent birthmarks or physical disabilities (reviewed in Oaten et al. 2011; Murray and Schaller 2016). People also tend to avoid individuals displaying signs of infectious illnesses, such as facial lesions (Murray and Schaller 2016). Because the costs of false-negative identification when assessing our environment for pathogens are likely greater than the costs of making false-positive identification ("the smoke detector principle," Nesse 2005), Oaten et al. (2011) proposed that both types of social avoidance (avoidance of individuals displaying signs of infectious illnesses and stigmatization of other types of individual, e.g., obese individuals)

Eugene J. Dawydiak

Eugene.Dawydiak@glasgow.ac.uk

1 School of Psychology, University of Glasgow, Glasgow Scotland, UK

2 Institute of Neuroscience \& Psychology, University of Glasgow, Glasgow, Scotland, UK reflect a common underlying mechanism: infectious disease avoidance. In other words, Oaten et al. (2011) suggested that the potentially "over inclusive" nature of avoidance of individuals displaying signs of infectious illnesses contributes to the stigmatization of other groups of atypical individuals (see also Kurzban and Leary 2001; van Leeuwen et al. 2015; Park et al. 2007).

To date, there have been relatively few direct empirical tests of Oaten et al.'s (2011) hypothesis. Park et al. (2007) found that concerns about infectious illnesses (assessed using the Perceived Vulnerability to Disease Scale, Park et al. 2003) were positively correlated with the extent to which both men and women stigmatized obese individuals. Similarly, Lieberman et al. (2012) found that scores on the pathogen disgust subscale of the Three Domain Disgust Scale (Tybur et al. 2009) were positively correlated with the extent to which people stigmatized obese individuals, although this pattern of the result was observed in women and not men. Positive links between scores on the Perceived Vulnerability to Disease Scale and stigmatization of individuals with non-infectious physical disabilities (e.g., leg amputations) have also been reported (Park et al. 2003). However, other work has implicated disgust in attitudes to homeless people and outgroup individuals, as well as political ideology 
(Brenner and Inbar 2014; Clifford and Piston 2017; van Leeuwen and Petersen 2018).

If Oaten et al. (2011) are correct that pathogen disgust contributes to individual differences in the stigmatization of atypical individuals, the association between pathogen disgust and stigmatization may not occur only for people with physical ailments. Indeed, overgeneralization of pathogen disgust might also contribute to the stigmatization of people with mental health conditions. To the best of our knowledge, this possibility has not yet been subject to a direct empirical test.

In light of the above, we investigated the possibility that overgeneralization of infectious disease avoidance similarly contributes to stigmatization of individuals with mental health conditions, here we investigated the relationships between each component of Tybur et al.'s (2009) Three Domain Disgust Scale (pathogen, sexual, and moral disgust) and individual differences in the stigmatization of individuals with three different mental health conditions: schizophrenia, skin picking disorder, and sexual sadism disorder.

\section{Methods}

One hundred and seventeen participants ( 26 men, 89 women, and two participants who chose not to report their sex; mean age $=24.08$ years, $\mathrm{SD}=9.09$ years; four participants chose not to report their age) took part in the study. Each participant completed Tybur et al.'s (2009) Three Domain Disgust Scale and the AQ-27 from Corrigan's toolkit for evaluating programs meant to erase the stigma of mental illness (Corrigan 2008). Participants were recruited from the University of Glasgow Psychology Participant Pool. With 117 participants, we have $80 \%$ power to detect $r=\sim .26$ (at alpha $=.05$ ).

The Three Domain Disgust Scale is a 21-item measure that asks participants to rate each of 21 actions from not at all disgusting (0) to extremely disgusting (6). The actions were divided into three domains: pathogen disgust (e.g., stepping on dog poop), sexual disgust (e.g., hearing two strangers having sex), and moral disgust (e.g., deceiving a friend). Scores on each subscale are summed and higher scores indicate greater disgust on that subscale. Mean scores and reliabilities for each subscale are given in Table 1.

The AQ-27 is a 27-item measure that assesses nine aspects of stigmatization (blame, anger, pity, help, dangerousness, fear, avoidance, segregation, and coercion) of an individual described in a vignette. Responses are made using a 1 (not at all) to 9 (very much) scale. Each participant completed this measure three times: once for an individual described as having either schizophrenia, or skin picking disorder, or sexual sadism disorder. Scores in response to each vignette were summed to indicate the overall extent to which the condition described was stigmatized. Vignettes were based on DSM-5 descriptions. Higher scores indicate greater stigmatization of
Table 1 Mean scores and reliabilities for each measure

\begin{tabular}{llll}
\hline & Mean & SD & Cronbach's alpha \\
\hline Pathogen disgust & 22.80 & 7.78 & .79 \\
Sexual disgust & 16.32 & 8.65 & .82 \\
Moral disgust & 25.68 & 7.51 & .81 \\
AQ-27 (schizophrenia) & 104.84 & 25.71 & .89 \\
AQ-27 (skin picking) & 80.69 & 22.66 & .87 \\
AQ-27 (sexual sadism) & 132.52 & 36.99 & .93 \\
\hline
\end{tabular}

that condition. Mean scores and reliabilities for each condition are given in Table 1.

The vignettes used in the study were:

"Peter has schizophrenia. Schizophrenia is a mental health disorder that presents with hallucinations, delusions and disorganized thinking. Peter believes he is being plotted against and watched by the government. $\mathrm{He}$ has poor grooming and hygiene; is bad at keeping eye contact and has little interest in socializing."

"Cathy suffers from skin picking disorder, also known as dermatillomania. This is where a person feels compelled to pick at their skin to the extent that they will cause visible, painful wounds. Cathy uses tweezers to excessively pick, squeeze and gouge at imagined skin deficits on her face. She frequently gets infections in these wounds but persists with the behavior."

"Bertie has been diagnosed with sexual sadism disorder. This is a paraphilic disorder characterized by the recurrent, intense sexual arousal from the physical or psychological suffering of another person. It manifests itself in fantasies, urges and behaviors."

\section{Results}

All data and analysis code are publicly available in the Open Science Framework (https://osf.io/aj9s6/). Analyses were conducted in R using lme4 (Bates et al. 2015) and lmerTest (Kuznetsova et al. 2017). Scores were $z$-scored prior to analyses.

We analyzed stigmatization using a mixed-effects model, with participant and vignette as random factors. Scores on the pathogen, sexual, and moral disgust subscales were entered simultaneously as predictors.

Our analysis showed a significant positive effect of pathogen disgust (estimate $=.265, \mathrm{SE}=.082, \mathrm{df}=86.9, t=3.22$, $p=.0018$ ). By contrast, the effects of moral disgust (estimate $=-.135, \mathrm{SE}=.084, \mathrm{df}=86.9, t=-1.62, p=.1089)$ and sexual disgust (estimate $=.113, \mathrm{SE}=.089, \mathrm{df}=86.9, t=$ $1.27, p=.207$ ) were not significant. Figure 1 shows the 

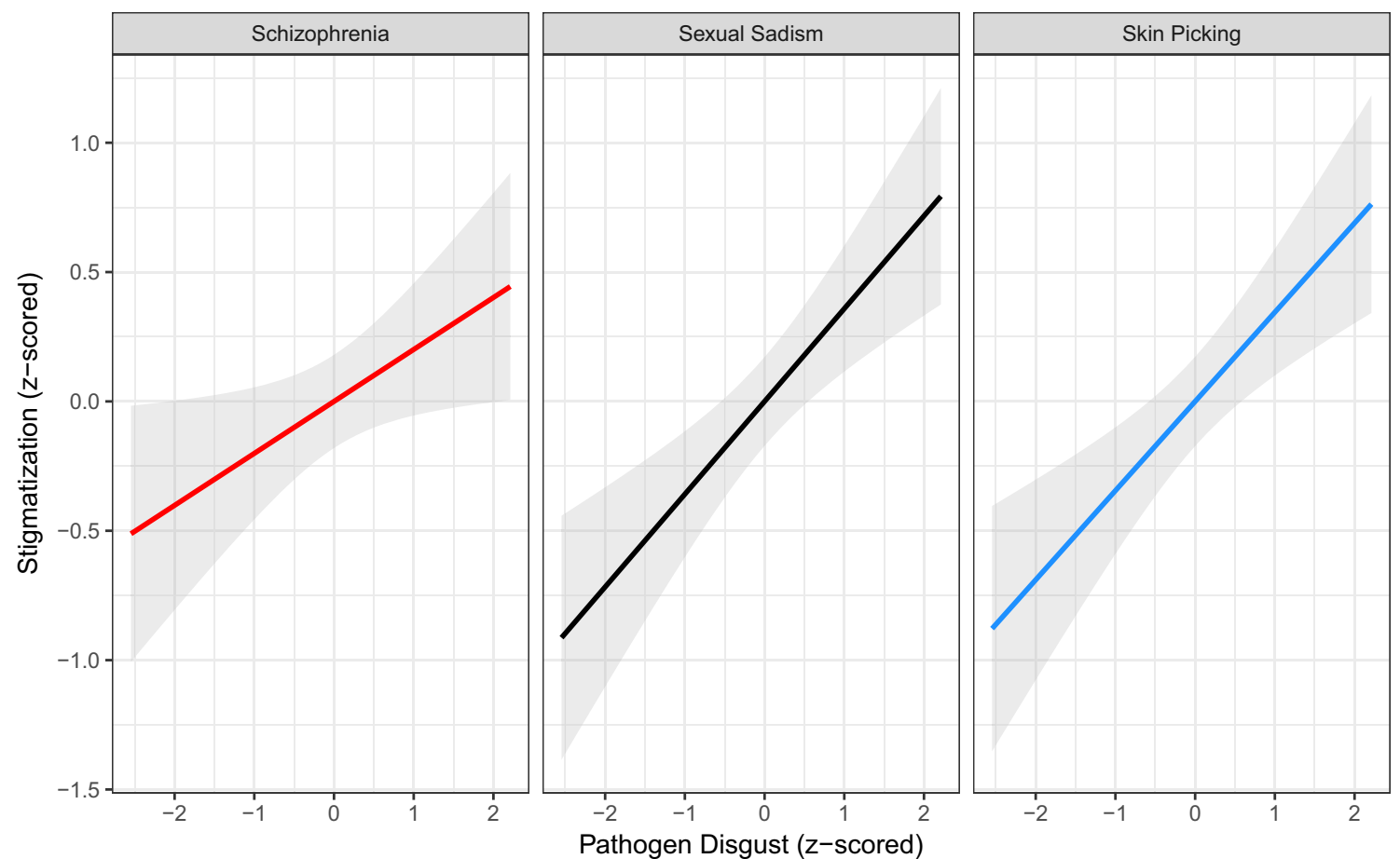

Fig. 1 The positive relationship between pathogen disgust and stigmatization of mental health conditions

relationship between pathogen disgust and stigmatization for each of the three mental health conditions. When analyzed separately, there were significant positive effects of pathogen disgust for skin picking and sexual sadism, but not schizophrenia (see https://osf.io/aj9s6/ for full analyses).

\section{Discussion}

The current study investigated possible relationships between components of disgust sensitivity and stigmatization of individuals with one of three mental health conditions (schizophrenia, skin picking disorder, or sexual sadism disorder). Results of a mixed-effects model showed a positive relationship between pathogen disgust and stigmatization of individuals with these mental health conditions. By contrast, neither sexual nor moral disgust was significantly related to the stigmatization of individuals with these mental health conditions in this analysis. When each mental health condition was analyzed separately, there were significant positive effects of pathogen disgust for skin picking and sexual sadism, but not schizophrenia. Collectively, these results are consistent with Oaten et al.'s (2011) hypothesis that the "over inclusive" nature of infectious disease avoidance contributes to the stigmatization of individuals with mental health conditions.

Although we find that pathogen disgust is positively correlated with the stigmatization of these three mental health conditions, the correlational study design we used in the current study does not allow us to conclude that pathogen disgust directly (i.e., causally) influences stigmatization of mental health conditions. Further work in which pathogen disgust is experimentally manipulated (following methods described in, e.g., Culpepper et al. 2018 or Tybur et al. 2011) would clarify this issue, although some recent work suggests that such priming manipulations do not necessarily have robust effects on social attitudes (see, e.g., Ji et al. 2019; Makhanova et al. 2019). Further work would also be needed to identify whether the observed association between pathogen disgust and stigmatization of individuals with the three mental health conditions examined in the current study also occurs for stigmatization of individuals with other mental health conditions. Such work could also include other control conditions (e.g., comparing effects of pathogen and violence threats) and disentangle potential correlations between mental health conditions, infectious illnesses, and other disease cues (i.e., address confounds between mental health symptoms and cues of infectiousness).

In conclusion, we find that pathogen disgust predicts the stigmatization of individuals with one of three mental health conditions (schizophrenia, skin picking disorder, and sexual sadism disorder). These results support the proposal that pathogen disgust and stigmatization of individuals with mental health conditions may reflect a common underlying motivation.

Acknowledgments The authors would like to thank Lisa DeBruine and Iris Holzleitner for help with analyses and Joshua Tybur and Stephanie Allan for constructive feedback on a draft of this manuscript. 
Data Availability Data and analysis code are publicly available at https:// osf.io/aj9s6/

Compliance with Ethical Standards The study was conducted in accordance with the British Psychological Society's Code of Ethics and Conduct.

Conflict of Interest The authors declare that they have no conflict of interest.

Open Access This article is distributed under the terms of the Creative Commons Attribution 4.0 International License (http:// creativecommons.org/licenses/by/4.0/), which permits unrestricted use, distribution, and reproduction in any medium, provided you give appropriate credit to the original author(s) and the source, provide a link to the Creative Commons license, and indicate if changes were made.

\section{References}

Bates, D., Maechler, M., Bolker, B., \& Walker, S. (2015). Fitting linear mixed-effects models using lme4. Journal of Statistical Software, 67(1), 1-48.

Brenner, C. J., \& Inbar, Y. (2014). Disgust sensitivity predicts political ideology and policy attitudes in the Netherlands. European Journal of Social Psychology, 45(1), 27-38.

Clifford, S., \& Piston, S. (2017). Explaining public support for counterproductive homelessness policy: the role of disgust. Political Behavior, 39(2), 503-525.

Corrigan, P. W. (2008). A toolkit for evaluating programs meant to erase the stigma of mental illness. Chicago: Illinois Institute of Technology.

Culpepper, P. D., Havlíček, J., Leongómez, J. D., \& Roberts, S. C. (2018). Visually activating pathogen disgust: a new instrument for studying the behavioural immune system. Frontiers in Psychology, 9, 1397.

Ji, T., Tybur, J. M., \& van Vugt, M. (2019). Generalized or origin-specific out-group prejudice?: the role of temporary and chronic pathogenavoidance motivation in intergroup relations. Evolutionary Psychology, 17(1), 1474704919826851.

Kurzban, R., \& Leary, M. R. (2001). Evolutionary origins of stigmatization: the functions of social exclusion. Psychological Bulletin, 127(2), 187-208.
Kuznetsova, A., Brockhoff, P. B., \& Christensen, R. H. B. (2017). lmerTest package: tests in linear mixed effects models. Journal of Statistical Software, 82(13), 1-26.

Lieberman, D. L., Tybur, J. M., \& Latner, J. D. (2012). Disgust sensitivity, obesity stigma, and gender: contamination psychology predicts weight bias for women, not men. Obesity, 20(9), 1803-1814.

Makhanova, A., Plant, E. A., Monroe, A. E., \& Maner, J. K. (2019). Binding together to avoid illness: pathogen avoidance and moral worldviews. Evolutionary Behavioral Sciences.

Murray, D. R., \& Schaller, M. (2016). The behavioral immune system: implications for social cognition, social interaction, and social influence. In Advances in experimental social psychology (Vol. 53, pp. 75-129). Academic Press.

Nesse, R. M. (2005). Natural selection and the regulation of defenses: a signal detection analysis of the smoke detector principle. Evolution and Human Behavior, 26(1), 88-105.

Oaten, M., Stevenson, R. J., \& Case, T. I. (2011). Disease avoidance as a functional basis for stigmatization. Philosophical Transactions of the Royal Society, B: Biological Sciences, 366(1583), 3433-3452.

Park, J. H., Faulkner, J., \& Schaller, M. (2003). Evolved diseaseavoidance processes and contemporary anti-social behavior: prejudicial attitudes and avoidance of people with physical disabilities. Journal of Nonverbal Behavior, 27(2), 65-87.

Park, J. H., Schaller, M., \& Crandall, C. S. (2007). Pathogen-avoidance mechanisms and the stigmatization of obese people. Evolution and Human Behavior, 28(6), 410-414.

Tybur, J. M., Lieberman, D., \& Griskevicius, V. (2009). Microbes, mating, and morality: individual differences in three functional domains of disgust. Journal of Personality and Social Psychology, 97(1), $103-122$.

Tybur, J. M., Bryan, A. D., Magnan, R. E., \& Hooper, A. E. C. (2011). Smells like safe sex: olfactory pathogen primes increase intentions to use condoms. Psychological Science, 22(4), 478-480.

van Leeuwen, F., \& Petersen, M. B. (2018). The behavioral immune system is designed to avoid infected individuals, not outgroups. Evolution and Human Behavior, 39(2), 226-234.

van Leeuwen, F., Hunt, D. F., \& Park, J. H. (2015). Is obesity stigma based on perceptions of appearance or character? Theory, evidence, and directions for further study. Evolutionary Psychology, 13(3), 1474704915600565 .

Publisher's Note Springer Nature remains neutral with regard to jurisdictional claims in published maps and institutional affiliations. 